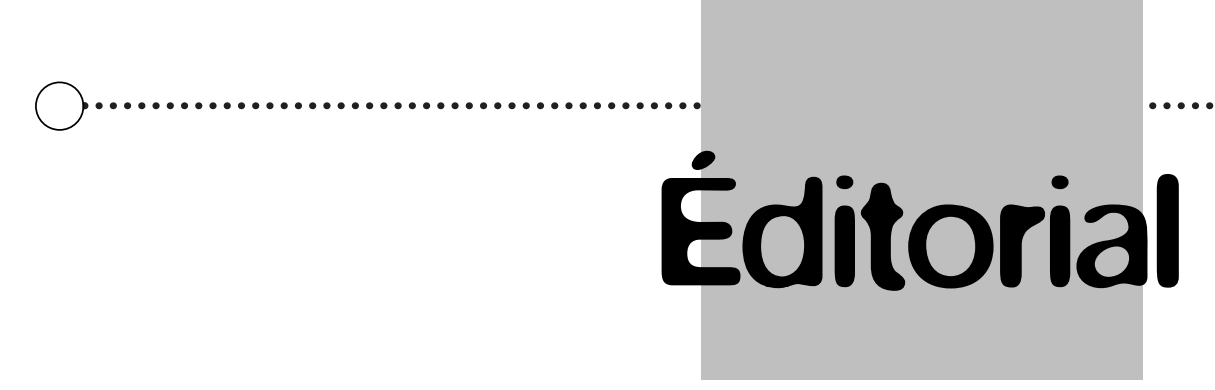

\title{
Quelle situation dans les universités de psychologie en 2007 ?
}

\section{What situation in the universities of psychology in 2007?}

\author{
Antoine BIOY*
}

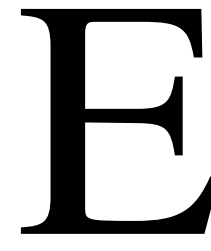

n France, ce n'est qu'en 1971 qu'un diplôme de « Psychologie Clinique » fut reconnu par les administrations de tutelle pour la première fois (à l'Université de Paris VII). Le titre quant à lui ne fut l'objet d'un cadre légal de pratique qu'un 1985. On voit combien la psychologie clinique, aux racines cependant anciennes, n'est en fait reconnu comme discipline à part entière que fort récemment. Elle mit en effet bien du temps avant de se détacher de ses deux racines principales, la philosophie et la médecine, pour affirmer sa singularité propre.

Près de 35 ans après ce premier diplôme universitaire institué, quelle est la situation actuelle ? On ne s'étonnera pas qu'elle suive en fait les turpitudes épistémologiques de sa cousine, la psychiatrie. Durant ces dernières années, deux batailles principales font rages, mais qui renvoient en fait à une même question.

La première bataille est celle qui concerne le terme de «psychologie clinique » : ce terme désigne-t-il toujours les seuls praticiens et enseignants chercheurs faisant l'hypothèse de l'inconscient? Ou inclut-il l'ensemble des psychologues qui suivent une méthodologie dite clinique (entretien, observation, tests et échelles, ou analyse de cas) ? Dans ce second cas, il faut alors y adjoindre notamment les psychologues cognitivo-comportementalistes, et les psychologues de la santé.

La seconde bataille, purement universitaire celle-ci, concerne l'enseignement de la psychopathologie. La grande majorité des enseignants chercheurs reconnaissent en effet que la psychologie clinique est indissociable de la psychopathologie, comme le seraient deux sœurs siamoises. Mais de quelle psychopathologie parle-t-on?

On retrouve, en réponse à cette question, la dichotomie devenue fort classique entre la psychopathologie dite a-théorique (dont l'emblème reste l'actuel DSM-IV) et la psychopathologie d'orientation psychanalytique. Les tenants de ces deux courants se livrent une guerre sans merci dans les lieux de savoirs; et dans le meilleur des cas, cette guerre se fait jusqu'au premier sang... 
Une illustration de cette guerre est actuellement la circulation sur Internet de deux pétitions. L'une émane des enseignants chercheurs d'orientation analytique («Sauvons la clinique »), et l'autre des partisans du courant dit a-théorique («Réformons la psychologie clinique française »). Chacun est le « mauvais objet» de l'autre et critique vertement le camp adverse. La psychopathologie psychanalytique serait passéiste, sans fondement scientifique réel, et les pratiques qui en découlent seraient non fondées et non pertinentes. La psychopathologie a-théorique ne reflèterait pas la réalité de la dynamique clinique, serait réductrice, et les méthodes qui s'y appuieraient seraient déshumanisantes voire non éthiques.

On assiste donc à une rivalité qui se nourrit du « contre»: être «contre» l'autre, pétition « contre » pétition, une pensée «contre » l'autre. Un comble pour une discipline, la psychologie, qui est censé évoluer en réfléchissant aux situations qu'elle rencontre! Actuellement, la situation dans les universités est sans nuance : il s'agit de rejoindre un camp et de lutter ! Une attitude qui, cela va sans dire, est non productive, pour le moins infantile, et surtout dangereuse pour son avenir (les instances ne savent plus à qui s'adresser, les terrains cliniques subissent ces guerres intestines, la recherche française peine à être reconnue, etc.).

$\mathrm{Au}$ milieu de tout cela, une troisième voie tente de se dégager, mais peine à voir le jour. Il s'agit des laboratoires universitaires qui préfèrent une approche plus intégrative. Ils sont cependant peu nombreux. Si l'on devait résumer cette «troisième voie », il s'agirait de n'exclure aucune approche qui, selon l'objet d'étude, le thème de la recherche, la pratique considérée, peut apporter un regard pertinent et le plus complet possible sur ce qui est observé. Par exemple, il est possible d'étudier le stress comme facteur de vulnérabilité psychique et dans un même temps, d'avoir une approche plus qualitative sur les évènements de vie et la dynamique intrapsychique des participants. Pour donner un autre exemple, cette voie psychopathologique permet à la fois de s'intéresser à ce qu'est un trouble obsessionnel compulsif (nomenclature DSM) tout en cherchant l'explication du TOC dans une compréhension psychodynamique des choses. On voit avec ces deux exemples que, finalement, le mouvement a-théorique et psychanalytique peuvent parfois être complémentaires. Mais à quel prix ? Sans doute un aménagement des deux courants. Il s'agit par exemple de ne pas s'enfermer dans les trois seuls modes de résolution de l'Edipe (névrose, psychose, perversion) mais de pouvoir entendre que d'autres nomenclatures peuvent parfois être plus parlantes pour aborder la clinique. Également, il s'agit de ne pas s'enfermer dans une seule étiopathogénie voulue scientifique, et d'accepter que la clinique puisse « déborder » les nomenclatures internationales et que rares sont les pathologies totalement «franches ». Il s'agit alors de penser l'autre, autrement, de façon plus qualitative et moins normée.

Il existe cependant un risque à cette « troisième voie ». Celle d'un syncrétisme opportuniste, d'une psychologie clinique un peu molle car voulant contenter chacun. Et c'est peutêtre là que l'université devrait « retourner» un peu plus dans les lieux de consultation, et ne pas rencontrer des patients qu'à visée de recherche (ce qui est le cas de nombres de nos collègues enseignant-chercheurs). L'université est trop souvent vue comme le lieu des recherches fondamentales ou cliniques qui viennent, ensuite, nourrir les pratiques cliniques. Or, le mouvement est bien transitif, et la clinique doit aussi pouvoir guider une évolution saine et dynamique de la psychopathologie moderne, hors cris et débats de chapelle. S'il est un avantage à l'organisation des facultés de médecine, c'est de laisser ses MCU-PH et PU$\mathrm{PH}$ au contact premier avec la pratique clinique. Dans les facultés de psychologie, au mieux peut-on quémander un $20 \%$ de temps en institution, et encore, ce temps est à justifier en tant que « terrain de recherche »...

Enfin, il serait impossible de terminer ce rapide panorama de la situation actuelle dans les universités de psychologie sans dire un mot de l'avenir. Car c'est l'enseignement prodigué en psychologie clinique et en psychopathologie aujourd'hui qui conditionne les pratiques de demain, via les futurs diplômés. Peut-on envisager qu'aujourd'hui un étudiant en psychologie clinique sorte des bancs de l'université sans savoir ce qu'est une «personnalité évitante » (il ne connaîtra que les névroses anxieuses) ou sans savoir ce que sont des "mécanismes de défense " (il ne connaîtra que le coping); sans qu'il fasse le lien entre le terme «paraphilie » et la théorie des perversions sexuelles selon Freud? C'est pourtant d'une privation de toute cette richesse dont il est question, lorsqu'un clan veut interdire l'autre... Les universités françaises saurontelle revenir vers une plus grande sagesse ?

Antoine Bioy

Secrétaire de rédaction Éditorialiste invité 